\title{
An Improved Technique for Profile Reconstruction of PEC Bodies
}

\author{
Yuri Álvarez, Senior Member, IEEE, María García-Fernández, Student Member, IEEE, Fernando Las- \\ Heras, Senior Member, IEEE
}

\begin{abstract}
This contribution describes an improved technique for model-based profile reconstruction of PEC objects. The technique takes advantage of the fact that the illuminated part of the OUT is, in general, accurately reconstructed when considering independent incident angles. The knowledge of the illuminated part is used to weight the reconstructed profile for each incidence. A comparison with the extended methodology that takes into account all the incident angles simultaneously is presented, proving that the proposed technique provides faster convergence to an accurate recovery of the OUT. Application examples considering 1 and 2 OUTs are presented.
\end{abstract}

Index Terms-Electromagnetic fields, Electromagnetic scattering, Inverse problems, Imaging.

\section{INTRODUCTION}

$\mathrm{E}$ LECTROMAGNETIC inverse scattering has been applied in different research fields such as medical imaging, non-destructive testing, security screening, and ground penetrating radar. The goal is to recover the geometry and/or the constitutive parameters of the scenario-under-test from the measured scattered or total field.

Among the great variety of inverse techniques developed in the last decades, it has been found that model-based profile reconstruction techniques are more efficient in the use of the available scattered field information than diffraction tomography [1] or inverse source methods [2]. Model-based profile reconstruction techniques relate the measured scattered field with the field scattered by an electromagnetic model of the Object-Under-Test (OUT) by means of a cost function, as in the flowchart depicted in Fig. 1 (a). The electromagnetic model of the OUT and the cost function optimization techniques are then the key points of model-based profile reconstruction. For example, the OUT can be parametrized using splines $[3,4]$ or Fourier series, and the scattered field can be calculated using a Method-of-Moments to solve the 2D integral equations [5]. Thus, the number of unknowns are just the coefficients, $\rho$, of the parametrized OUT profile.

Manuscript received March 10, 2016. This work has been supported by the "Ministerio de Economía y Competitividad" of Spain/FEDER under project TEC2014-54005-P (MIRIIEM); and by the "Gobierno del Principado de Asturias" under project GRUPIN14-114.

Y. Álvarez, María García-Fernández, and Fernando Las-Heras are with the Área de Teoría de la Señal y Comunicaciones, Universidad de Oviedo. Edificio Polivalente, Módulo 8, Campus Universitario de Gijón. E-33203, Gijón (Spain). Phone: +34 686404 088. Fax: +34 985182 466. Email: yalopez@tsc.uniovi.es ; mariagarcia@tsc.uniovi.es ; flasheras@tsc.uniovi.es.
Optimization techniques, such as Genetic Algorithms (GA) $[1,6]$, Particle Swarm Optimization (PSO) [4,6], or Differential Evolution [3,6], have been successfully used for cost function minimization.

The main drawback of model-based profile reconstruction techniques is the increase of the computational complexity associated to the forward model used to calculate the scattered field of the parametrized OUT. Thus, this contribution proposes a technique to improve model-based techniques convergence by means of a new optimization strategy.

\section{Profile Reconstruction Method}

Most of the model-based techniques use spatial diversity to improve the accuracy of the reconstruction, illuminating the OUT under multiple incidences, $K$. For each $k$-th incidence, the scattered field is acquired at $N$ observation points, $E_{\text {scatt,n,k,nmeas }}$, and compared with the field scattered by an electromagnetic model of the OUT, $E_{\text {scatt,n,ksim }}(\rho)$. In this work, a 2D Method-of-Moments formulation for TM polarization is considered [5]. A detailed integral equation model-based formulation for 2D PEC OUTs is presented in $[3,4]$. The cost function to be minimized, $F(\rho)$ (Fig. 1 (a)), combines all the incidences and observation points $[3,4,6]$. At the beginning, an initial population of Npop candidates is created. Each candidate within the population is a set of OUT parametrization coefficients. After minimization, the candidate associated to the minimum cost function value, $\rho_{\text {best, }}$ is chosen as the best solution for the given profile reconstruction problem (Fig. 1 (a)).

In order to improve the convergence of the optimization techniques, and then, reducing the calculation time, multistage strategies have been studied. For example, in [7] a 2stage method is proposed. In the first stage, depicted in black boxes in Fig. 1 (b), a cost function $F_{\mathrm{k}}(\rho)$ is minimized for each $k$-th incidence. Best candidates $\rho_{\mathrm{k}, \text { best }}$ are considered in the initial population of the second stage, where the cost function $F(\rho)$, Fig. 1 (a), is minimized.

In the case of PEC objects, it has been found that the first stage is efficient in recovering the part of the OUT profile illuminated by the incident field. Then, it could be of interest to discard the recovered OUT profile that is not illuminated by the $k$-th incident field. Additionally, such part of the OUT profile illuminated by the incident field, typically presents the highest amplitude values of the induced currents.

With these previous considerations, the profile 
reconstruction technique depicted in Fig. 1 (b) can be improved by adding the physics analysis that takes into account: i) the OUT profile illumination, $\left(\left(1-\hat{k}_{\text {inc }} \bullet \hat{n}\right) / 2\right.$ , where $\hat{k}_{i n c}$ is the Poynting vector of the incident field, and $\hat{n}$ the OUT profile normal vector), and ii) the normalized amplitude of the induced currents. The product of these two quantities (see Fig. 2) gives the weighting coefficients, $W_{\mathrm{k}}$ (Fig. 2). Best candidates for every $k$-th incidence are then combined according to the weighting coefficients $W_{\mathrm{k}}$ (Fig. 1 (b), red boxes), obtaining the best candidate under such considerations, $\rho_{\text {comb }}$.

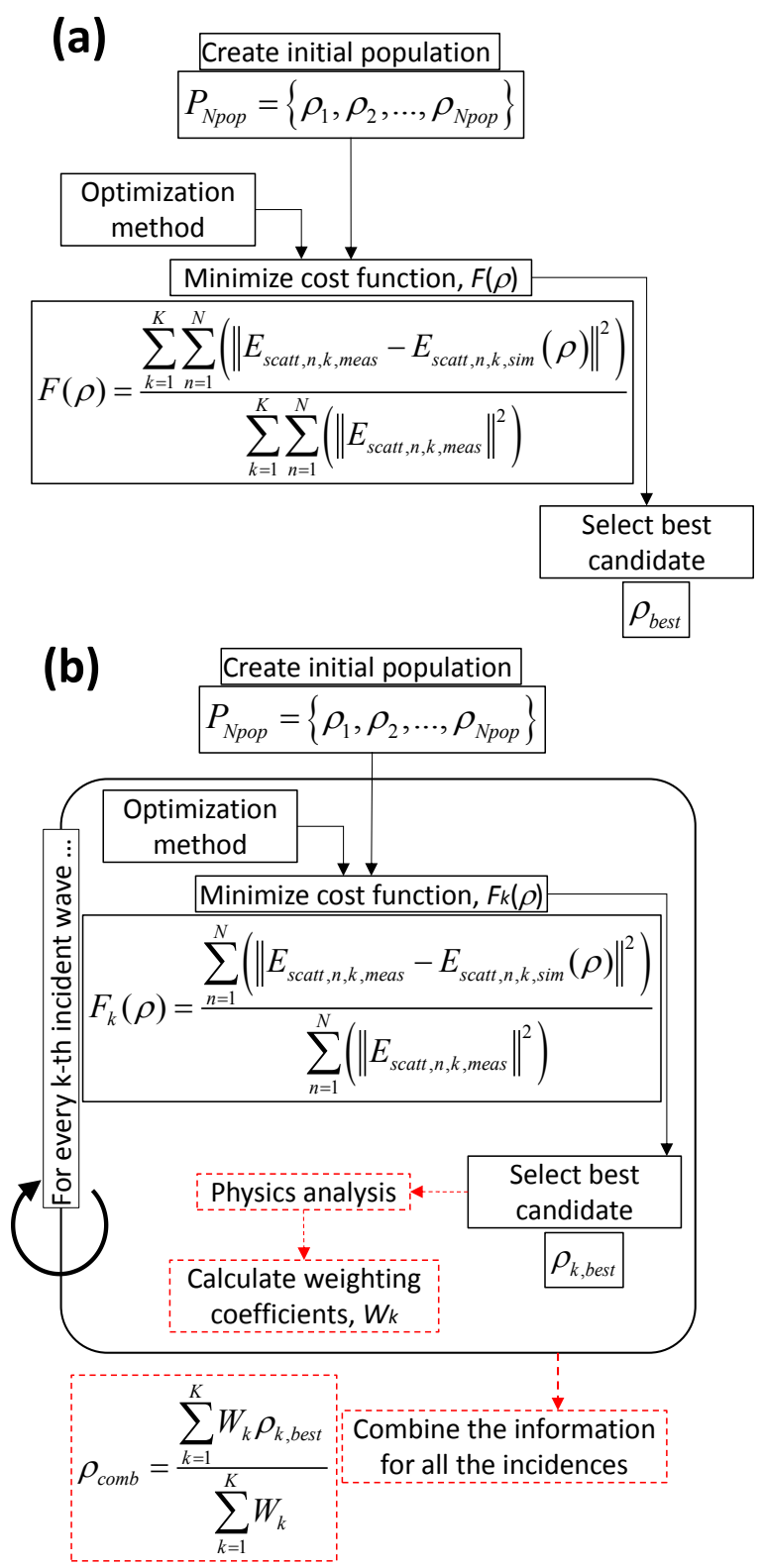

Fig. 1. Flowchart of the two compared methods. (a) Considering all the $K$ incident directions together. (b) Incident directions are processed independently. Physics processing (dashed red boxes and arrows) is applied to improve convergence.

\section{APPLICATION EXAMPLE}

Model-based profile reconstruction techniques depicted in
Fig. 1 have been compared aiming to prove that the consideration of the induced currents and the incident field illumination can improve the convergence. A basic PSO method, with inertial weight and correction factor coefficients set to 1 and 2, respectively, and a population size of 50 candidates, has been selected for cost function minimization. Pchip splines have been selected as they allow accurate parameterization of fast-varying contours with aspect angle, using a reduced number of control points (red dots in Fig. 2) $[3,4]$, that can be parameterized in polar coordinates. If the control points are equally spaced in $\phi$, then the only unknowns are the radii of the control points, $\rho_{\mathrm{cp}}$ (Fig. 2) together with the center of the parameterized out, $x_{\mathrm{c}}, y_{\mathrm{c}}$, i.e. $\rho=\left[\rho_{\mathrm{cp}} x_{\mathrm{c}} y_{\mathrm{c}}\right]$.

Furthermore, this parameterization can be easily extended to two or more OUTs by expanding the unknowns vector: $\rho=$ [ $\rho_{\mathrm{cp}, \text { OUT1 } 1} x_{\mathrm{c}, \text { OUT1 }} y_{\mathrm{c}, \text { OUT1 }} \rho_{\mathrm{cp}, \text { OUT2 }} x_{\mathrm{c}}$, OUT2 $y_{\mathrm{c}, \text { OUT2 }} \ldots$ ].

Reconstruction accuracy depends on the amount of available information: number of incident directions $\left(N_{\text {inc }}\right)$, number of acquisition points, and also the OUT electric size (since it influences the scattered field variation with respect to the aspect angle). For this example, point source transmitters, placed in a $R_{\mathrm{Tx}}=75 \mathrm{~cm}$ circumference, are considered. The scattered field is acquired in a $R_{\mathrm{Rx}}=30 \mathrm{~cm}$ circumference, sampled every $4^{\circ}$ (resulting in 90 acquisition points) to ensure that the Nyquist sampling rate is fulfilled [8]. OUT size is also analyzed for sizes ranging from $0.5 \lambda$ to $3 \lambda$. Noise has been added to the scattered field according to a SNR of $30 \mathrm{~dB}$.

Imaging domain size is $20 \times 20 \mathrm{~cm}$ (see Fig. 7), which is larger than the maximum size of the OUT estimated from the analysis of the scattered field spectral bandwidth [8]. The unknowns search space is set to $\rho \in[0.57] \mathrm{cm}$ to ensure that the initial population candidates are randomly spread across the imaging domain. The initial population set, $P_{\text {Npop }}$, is created according to a uniform probability distribution fitting this search space interval. Concerning the number of unknowns, for this example $N_{\rho}=14$ has been chosen, that is, $N \rho_{\mathrm{cp}}=12$ control points, plus $x_{\mathrm{c}}, y_{\mathrm{c}}$ ( 2 unknowns).

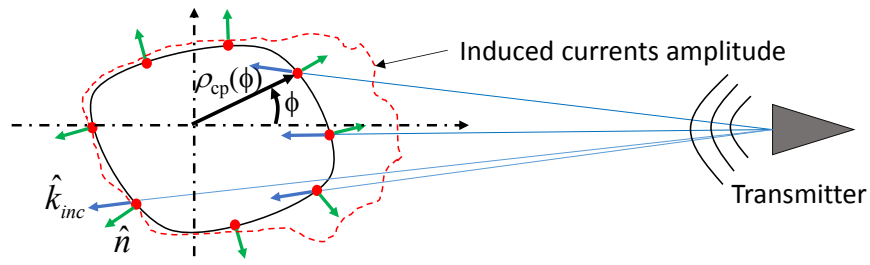

Fig. 2. Scheme of physics processing for weighting the reconstructed profile. Illumination of the OUT profile and induced currents amplitude are considered.

Aiming to highlight the fact that the parts of the OUT illuminated by the incident field can be accurately recovered, Fig. 3 shows the reconstruction results for two incident directions $\left(\phi_{\text {inc }}=-90^{\circ}\right.$, Fig $3(\mathrm{a})$, and $\phi_{\text {inc }}=0^{\circ}$, Fig. 3 (b)). Note that the highest amplitude of the induced currents takes place in the illuminated part of the OUT. 

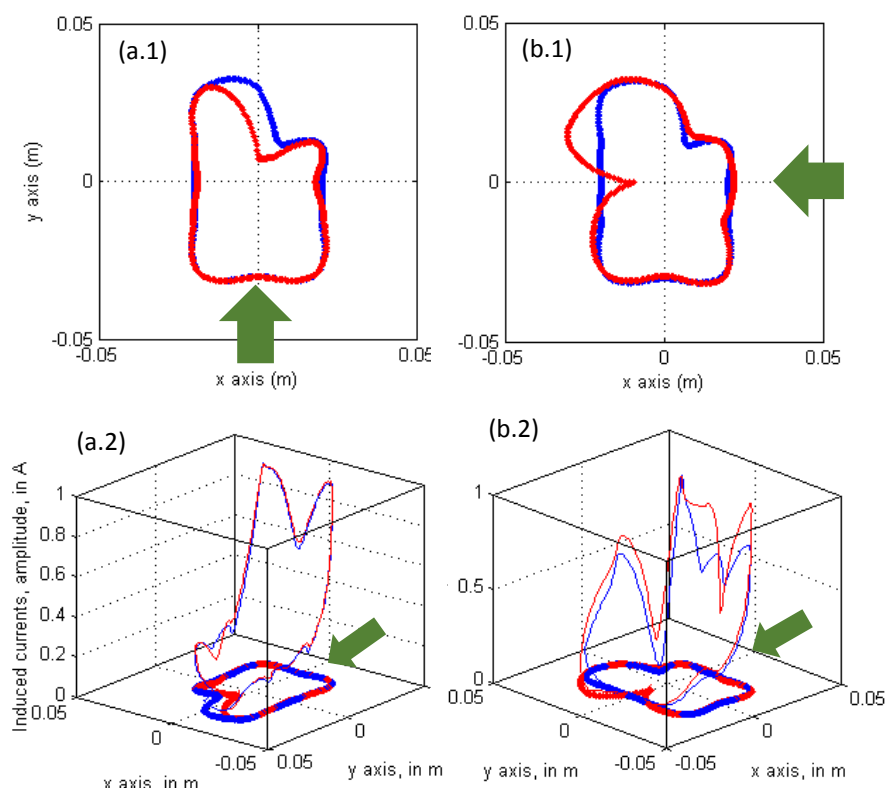

Fig. 3. (a.1) and (b.1): true profile (blue) and reconstructed profile (red) considering the $k$-th incident direction denoted with the green arrow. (a.2) and (b.2): induced currents on the true (blue) and reconstructed (red) OUT profile for the $k$-th incident direction. OUT size: $1.9 \lambda$.
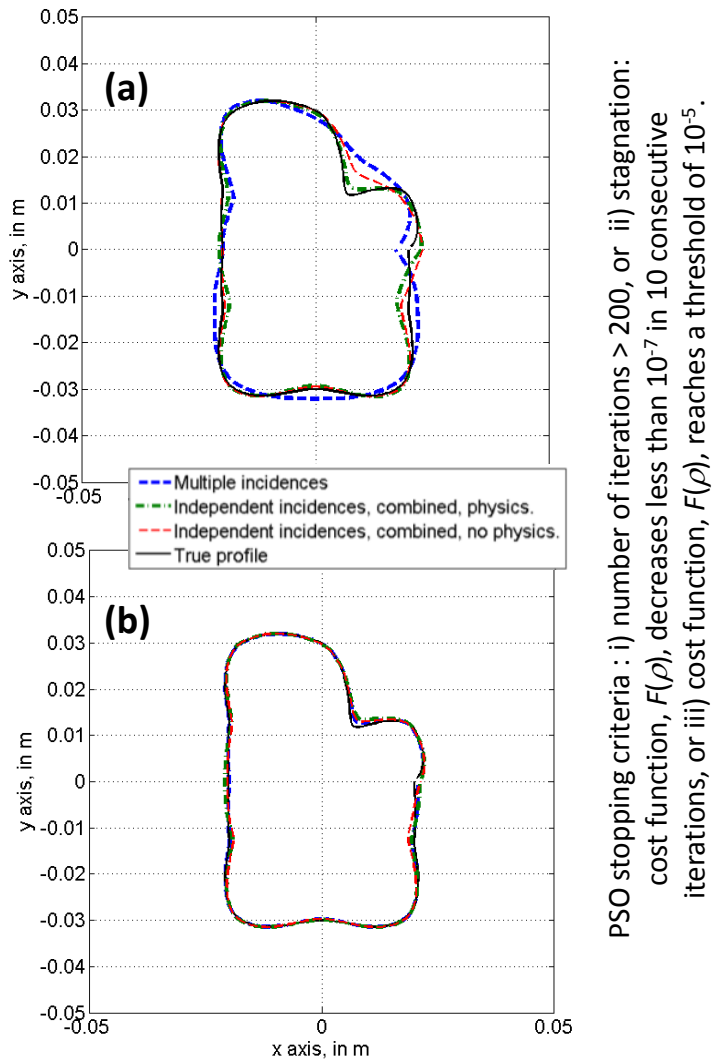

Fig. 4. True and reconstructed OUT profile for the compared inversion techniques depicted in Fig. 1. $N_{\text {inc }}=4$. (a) $D_{\text {out }}=0.5 \lambda$. (b) $D_{\text {out }}=1 \lambda$.

To compare the convergence of the developed techniques, the number of PSO iterations for a target reconstruction error $\varepsilon$, is analyzed. This metric is more precise than the cost function value, $F(\rho)$, as it will be shown that, for electrically small OUTs significant variations in the OUT profile do not have a noticeable impact in the scattered field. The reconstruction error $\varepsilon$ between the true and recovered OUT profile is:

$$
\varepsilon=100 \times\left|\rho_{\text {true }}(\phi)-\rho_{\text {rec }}(\phi)\right| / \rho_{\text {true }}(\phi)
$$

Reconstruction results for different OUT sizes $\left(D_{\text {out }}\right.$, in $\left.\lambda\right)$ and number of transmitters, $N_{\text {inc }}$ ( 4 and 8 , equally spaced in the $\mathrm{R}_{\mathrm{Tx}}=75 \mathrm{~cm}$ circumference) are summarized in Table I. Two target reconstruction errors, $\varepsilon=5 \%$ and $\varepsilon=2 \%$, are considered. For OUT resonant dimensions (around 1-2 $\lambda$ ), model-based reconstruction techniques reach $\varepsilon=5 \%$ in less than 130 iterations. Furthermore, for $N_{\text {inc }}=8$, if the best candidates for each incidence are combined, $\rho_{\mathrm{k}, \text { best }}$ ('Indep. incid., combined, physics'), convergence is achieved in much less iterations than when all the incidences are considered ('Multiple incidences'), see Fig. 5. For $N_{\text {inc }}=4$, both techniques require similar number of iterations to reach $\varepsilon=5 \%$.

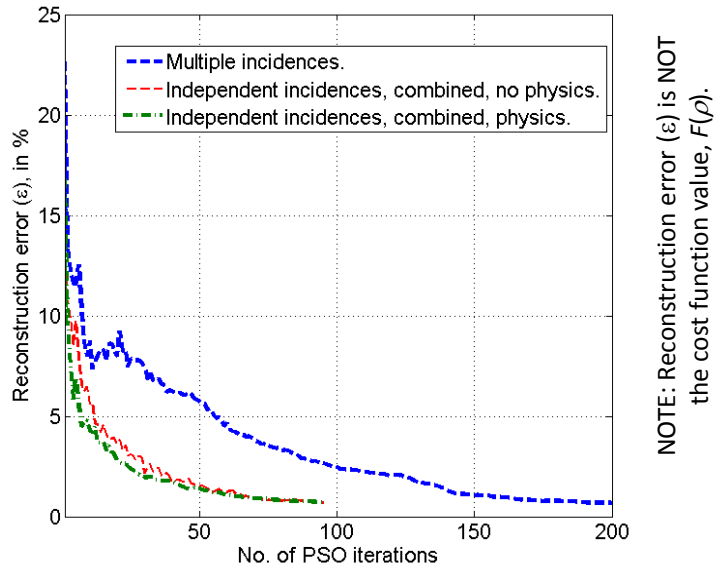

Fig. 5. Convergence of the compared inversion techniques depicted in Fig. 1. $N_{\text {inc }}=8 . D_{\text {out }}=1 \lambda$.

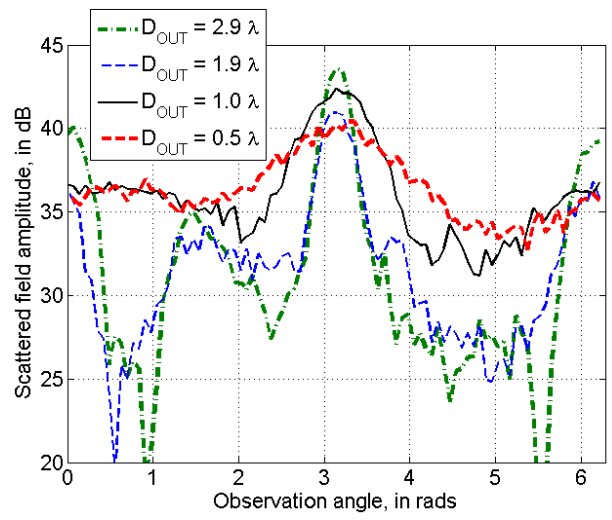

Fig. 6. Amplitude of the field scattered by the OUT depicted in Fig. 4 as a function of the electric size $\left(D_{\text {out }}\right)$. SNR $=30 \mathrm{~dB}$.

The scattered fields of electrically large OUTs have fastaspect angle-variation behavior fields (Fig. 6), creating several local minima in the cost function where global optimization methods can be trapped [6]. On the other side, the scattered field of electrically small OUTs has smooth variation, so slightly different profiles will scatter similar slow-aspect angle-varying scattered fields. In consequence, model-based profile reconstruction techniques are expected to have worse 
performance for extreme cases of the electrical size (very large or very small) of OUT, as shown in Table I.

TABLE I

PSO ITERATIONS REQUIRED FOR A TARGETED RECONSTRUCTION ERROR

\begin{tabular}{|c|c|c|c|c|c|c|c|}
\hline \multirow[t]{2}{*}{$N_{\text {inc }}$} & \multirow[t]{2}{*}{$\begin{array}{l}D_{\text {out }} \\
(\lambda)\end{array}$} & \multicolumn{2}{|c|}{$\begin{array}{c}\text { Multiple } \\
\text { incidences }\end{array}$} & \multicolumn{2}{|c|}{$\begin{array}{c}\text { Indep. incid., } \\
\text { combined, } \\
\text { no physics }\end{array}$} & \multicolumn{2}{|c|}{$\begin{array}{c}\text { Indep. incid., } \\
\text { combined, } \\
\text { physics }\end{array}$} \\
\hline & & $\varepsilon<5 \%$ & $\varepsilon<2 \%$ & $\varepsilon<5 \%$ & $\varepsilon<2 \%$ & $\varepsilon<5 \%$ & $\varepsilon<2 \%$ \\
\hline \multirow{4}{*}{4} & 0.5 & 70 & $>200$ & 25 & 164 & 14 & 38 \\
\hline & 1.0 & 21 & 33 & 19 & 56 & 19 & 38 \\
\hline & 1.9 & 14 & 24 & 22 & 45 & 18 & 20 \\
\hline & 2.9 & \multicolumn{2}{|c|}{ Stagn., $\varepsilon>16 \%$} & \multicolumn{2}{|c|}{ Stagn., $\varepsilon>9 \%$} & \multicolumn{2}{|c|}{ Stagn., $\varepsilon>7 \%$} \\
\hline \multirow{4}{*}{8} & 0.5 & 94 & $>200$ & 43 & $>200$ & 14 & $>200$ \\
\hline & 1.0 & 56 & 125 & 12 & 40 & 7 & 30 \\
\hline & 1.9 & 17 & 25 & 7 & 42 & 12 & 18 \\
\hline & 2.9 & 28 & 29 & Stagn & $>10 \%$ & Stagn & $\varepsilon>6 \%$ \\
\hline
\end{tabular}

Stagn.: stagnation. $\varepsilon$ : reconstruction error. 1 OUT.

TABLE II

PSO ITERATIONS REQUIRED FOR A TARGETED RECONSTRUCTION ERROR

\begin{tabular}{c|cc|cc|cc}
\hline \hline \multirow{2}{*}{$\begin{array}{c}\text { SNR } \\
(\mathrm{dB})\end{array}$} & \multicolumn{2}{|c|}{$\begin{array}{c}\text { Multiple } \\
\text { incidences }\end{array}$} & \multicolumn{2}{|c|}{$\begin{array}{c}\text { Indep. incid., } \\
\text { combined, } \\
\text { no physics }\end{array}$} & \multicolumn{2}{|c}{$\begin{array}{c}\text { Indep. incid., } \\
\text { combined, } \\
\text { physics }\end{array}$} \\
\cline { 2 - 7 } & $\varepsilon<5 \%$ & $\varepsilon<2 \%$ & $\varepsilon<5 \%$ & $\varepsilon<2 \%$ & $\begin{array}{c}\varepsilon<5 \% \\
\varepsilon<2 \%\end{array}$ \\
\hline 30 & 21 & 33 & 19 & 56 & 19 & 38 \\
\hline 20 & 27 & $\begin{array}{c}\text { Stagn. } \\
\varepsilon>2.8 \%\end{array}$ & 18 & $\begin{array}{c}\text { Stagn. } \\
\varepsilon>4.5 \%\end{array}$ & 20 & 91 \\
\hline 10 & 29 & $\begin{array}{c}\text { Stagn. } \\
\varepsilon>4.5 \%\end{array}$ & \multicolumn{2}{|c|}{$\begin{array}{c}\text { Stagn. } \\
\varepsilon>7.8 \%\end{array}$} & \multicolumn{2}{|c|}{$\begin{array}{c}\text { Stagn. } \\
\varepsilon>6.2 \%\end{array}$} \\
\hline \hline
\end{tabular}

Stagn.: stagnation. $\varepsilon$ : reconstruction error. Ninc: 4.1 OUT, $D_{\text {out }}: 1.0 \lambda$

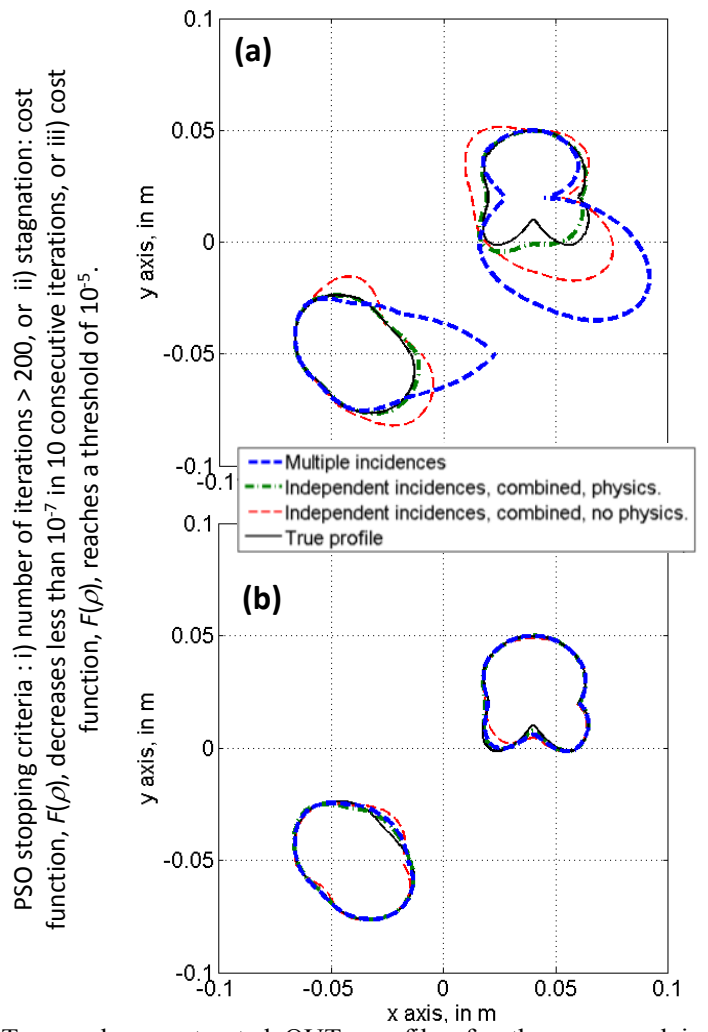

Fig. 7. True and reconstructed OUTs profiles for the compared inversion techniques depicted in Fig. 1. $N_{\text {inc }}=4$. (a) $D_{\text {out }}=2.8 \lambda$. (b) $D_{\text {out }}=1.2 \lambda$.

The influence of the noise in the compared techniques has also been analyzed. As shown in Table II, the technique 'Indep. incid., combined, physics' performs better for higher SNR. For lower SNR, compared methods have similar performance, resulting in PSO stagnation with a reconstruction error $\varepsilon>2 \%$.

TABLE III

PSO ITERATIONS REQUIRED FOR A TARGETED RECONSTRUCTION ERROR

\begin{tabular}{c|cc|cc|cc}
\hline \hline \multirow{2}{*}{$\begin{array}{c}D_{\text {out }} \\
(\lambda)\end{array}$} & \multicolumn{2}{|c|}{$\begin{array}{c}\text { Multiple } \\
\text { incidences }\end{array}$} & \multicolumn{2}{|c|}{$\begin{array}{c}\text { Indep. incid., } \\
\text { combined, } \\
\text { no physics }\end{array}$} & \multicolumn{2}{|c}{$\begin{array}{c}\text { Indep. incid., } \\
\text { combined, } \\
\text { physics }\end{array}$} \\
\cline { 2 - 7 } & $\varepsilon<5 \%$ & $\varepsilon<2 \%$ & $\varepsilon<5 \%$ & $\varepsilon<2 \%$ & $\varepsilon<5 \%$ & $\varepsilon<2 \%$ \\
\hline 0.6 & 4 & 61 & 5 & $\begin{array}{c}\text { Stagn., } \\
\varepsilon>2.8 \%\end{array}$ & 13 & 73 \\
\hline 1.2 & 8 & 16 & 3 & 52 & 2 & 16 \\
\hline 1.8 & 3 & 21 & 11 & 35 & 10 & 31 \\
\hline 2.8 & \multicolumn{2}{|c|}{ Stagn., $\varepsilon>9 \%$} & \multicolumn{2}{|c|}{ Stagn., $\varepsilon>5.7 \%$} & 21 & $\begin{array}{c}\text { Stagn., } \\
\varepsilon>2.8 \%\end{array}$ \\
\hline \hline
\end{tabular}

Stagn.: stagnation. $\varepsilon$ : reconstruction error. 2 OUTs.

Finally, the performance of the compared techniques has been tested for multiple OUTs. In this case, a larger number of unknowns is required, $N_{\rho}=20$, that is $N \rho_{\mathrm{cp}, \text { OUT1 }}=N \rho_{\mathrm{cp}, \text { OUT2 }}=8$ control points, plus $x_{\mathrm{c}}, y_{\mathrm{c}}$ of each OUT. Reconstruction results are summarized in Table III and depicted in Fig. 7. In this case, the 'Indep. incid., combined, physics' technique has better performance than the conventional method ('Multiple incidences') for the case of the OUT with largest electric size.

\section{CONCLUSIONS}

The use of the incident field illumination and the induced currents on the OUT profile has been proved to be quite effective to improve the convergence of model-based profile reconstruction techniques, with respect to the extended method based on a cost function relating all the incident directions simultaneously. Further analysis will be devoted to extent this study to dielectric objects, using electric and magnetic equivalent currents.

\section{REFERENCES}

[1] Marklein, R., Balasubramanian, K., Qing, A., \& Langenberg, K. J. "Linear and nonlinear iterative scalar inversion of multi-frequency multi-bistatic experimental electromagnetic scattering data," Inverse Problems, vol. 17, no. 6, pp. 1597-1610, 2001.

[2] López, Y. A., Domínguez-Casas, A., García-González, C., \& Las-Heras, F. "Geometry reconstruction of metallic bodies using the sources reconstruction method," IEEE Antennas and Wireless Propagation Letters, Vol. 9, pp. 1197-1200.

[3] Qing, A. "Electromagnetic inverse scattering of multiple twodimensional perfectly conducting objects by the differential evolution strategy," IEEE Transactions on Antennas and Propagation, Vol. 51, No. 6, pp. 1251-1262, 2003.

[4] Rekanos, I. T., "Shape reconstruction of a perfectly conducting scatterer using differential evolution and particle swarm optimization," IEEE Transactions on Geoscience and Remote Sensing, Vol. 46, No. 7, pp. 1967-1974, 2008

[5] R. F. Harrington, Field Computation by Moment Methods. Piscataway, NJ: IEEE Press, 1993.

[6] Rocca, P., Oliveri, G., \& Massa, A, "Differential evolution as applied to electromagnetics," IEEE Antennas and Propagation Magazine, Vol. 53, No. 1, pp. 38-49, 2011.

[7] Garcia-Fernandez, M., Garcia, C., Alvarez, Y, \& Las-Heras, F., "Influence of contour smoothness and electric size on the profile reconstruction of metallic objects using hybrid optimization," in EUROCON 2015, IEEE International Conference on Computer as a Tool, Salamanca (Spain), 8-11 September 2015, pp.1-6.

[8] Bucci, O.M., Franceschetti, G., "On the degrees of freedom of scattered fields," IEEE Transactions of Antennas and Propagation, Vol. 37, No. 7, pp. 918-926, 1989. 